\title{
KSHV regulation of fibulins in Kaposi's sarcoma: implications for tumorigenesis
}

\author{
Donald Alcendor ${ }^{1 *}$, Susan Knobel', Wen Zhu1', Prashant Desai ${ }^{2}$, Gary Hayward² \\ From $12^{\text {th }}$ International Conference on Malignancies in AIDS and Other Acquired Immunodeficiencies \\ (ICMAOI) \\ Bethesda, MD, USA. 26-27 April, 2010
}

Kaposi's sarcoma (KS) is an angioproliferative tumor of vascular endothelial cells and produces rare B cell lymphoproliferative diseases in the form of primary effusion lymphomas (PELs) and some forms of Multicentric Castleman's Disease (MCD). Kaposi's sarcoma-associated herpesvirus, also known as KSHV or human herpesvirus type 8 (HHV8), is the etiological agent of KS. Fibulins are extra-cellular matrix (ECM) proteins involved in cell adhesion, proliferation, migration, invasion, and angiogenesis, and have been linked to progression of several cancer types. However, to our knowledge, they have not been studied in KS. We examined fibulin-2 because we found it to be significantly downregulated by microarray analysis in KSHV-infected DMVEC cells. Here we demonstrate that fibulin-2 protein expression is downregulated 50-fold in 10 day KSHV-infected dermal microvascular endothelial cells (DMVEC) with a 26-fold reduction in fibulin-2 message. By time-course transcriptional analysis there was consistent reduction of fibulin-2 message accompanied by an increase in KSHV latency associated nuclear antigen (LANA) transcription. Of the fibulins assayed, fibulins $-2,-5$, and -3 were downregulated over time in KSHV infected DMVEC, while fibulins $1 \mathrm{C}$ and $1 \mathrm{D}$ were upregulated, with no change in fibulins 4, 6, and 7. In pleural effusion lymphoma cell lines that express different levels of KSHV lytic replication, we observed no detectable fibulin-2 expression. Tissue microarrays representing patch/plaque and nodular forms of KS from 86 different patients were shown to be statistically significant for downregulation of fibulin-2 in virus-infected LANA positive cells by dual labeled immunohistochemical staining. This represents the first study that examines fibulin-2 expression in KSHV-infected DMVEC and KS to determine whether suppression of this ECM protein plays a role in KS tumor progression. Understanding the interactions between KSHV and the fibulin family of extra-cellular matrix proteins that modulate angiogenesis cancer cell proliferation, migration and invasion could lead to development of novel therapies for treatment of KS.

\section{Acknowledgements}

This article has been published as part of Infectious Agents and Cancer Volume 5 Supplement 1, 2010: Proceedings of the $12^{\text {th }}$ International Conference on Malignancies in AIDS and Other Acquired

Immunodeficiencies (ICMAOI). The full contents of the supplement are available online at http://www.biomedcentral.com/1750-9378/5? issue=\$1

\section{Author details}

${ }^{1}$ Meharry Medical College, School of Medicine, Center for AIDS Health Disparities Research and Department of Microbiology and Immunology, Nashville, TN, USA. ²Department of Viral Oncology and Pharmacology, Molecular Virology Laboratories, Sidney-Kimmel Cancer Research Center, Johns Hopkins University School of Medicine, Baltimore, MD, USA.

Published: 11 October 2010

doi:10.1186/1750-9378-5-S1-A50

Cite this article as: Alcendor et al.: KSHV regulation of fibulins in Kaposi's sarcoma: implications for tumorigenesis. Infectious Agents and Cancer 2010 5(Suppl 1):A50.

*Correspondence: dalcendor@mmcedu

${ }^{1}$ Meharry Medical College, School of Medicine, Center for AIDS Health Disparities Research and Department of Microbiology and Immunology, Nashville, TN, USA

Full list of author information is available at the end of the article 